Radial and Nonradial Pulsations as Probes of Stellar Physics

ASP Conference Series, Vol. 259, 2002

C. Aerts, T.R. Bedding, \& J. Christensen-Dalsgaard, eds.

\title{
Differential Time-Series Photometry of BL Camelopardalis: Revisited
}

\author{
Chulhee Kim \\ Department of Earth Science Education, Chonbuk National University, \\ Korea \\ Y.B.- Jeon, S.-L. Kim \\ Korea Astronomy Observatory, Yeoungcheon 770-880, Korea
}

\begin{abstract}
New differential time-series observations of the white dwarf BL Camelopardalis were obtained using the B and V filters. It was confirmed that BL Cam is a double star but the effect of the secondary star on the periods is almost negligible. The (O-C) diagram and periods were analyzed.
\end{abstract}

We have undertaken differential CCD photometric observations of BL Cam to investigate its multiperiodicity and pulsational characteristics. Because we found a possibility that BL Cam is a double star from our previous CCD images with good seeing (Kim \& Sim, 1999), we observed BL Cam with a 1.8m telescope for better resolution.

A total number of 460 and 70 frames for $\mathrm{V}$ and $\mathrm{B}$ filters were obtained. We found that $\mathrm{BL}$ Cam is not a single star but a double star. The reason why this fact has not been confirmed is that small size telescopes, less than about $1 \mathrm{~m}$ class, have been used and the secondary could not be resolved. As far as we know, this is the first observation of BL Cam with a $1.8 \mathrm{~m}$ telescope. Therefore we applied the PSF photometric reduction procedure in order to eliminate the effect of the secondary star. It was confirmed that the effect of the secondary star on the periods is almost negligible.

The new times of maximum light together with those from the literature are plotted in Fig. 1 against the number of cycles E elapsed from the initial epoch of the ephemeris provided by McNamara \& Feltz (1978). We can see that the period variation was recently reversed. A linear ephemeris determined through a least-squares fit for 240 maximum times gives

$$
\begin{aligned}
\mathrm{HJD}_{\max }= & 2443124.855157+0.039103180 \cdot E . \\
& \pm 0.062890 \pm 0.000000345
\end{aligned}
$$

By applying the traditional method of Fourier transformation for period search, we determined a total of nine frequencies. Table 1 summarizes the coefficients of the synthetic light curves. We are now investigating the pulsation modes using the phase difference of $\phi_{V}-\phi_{B-V}$ as well as $A_{B-V} / A_{V}$. 


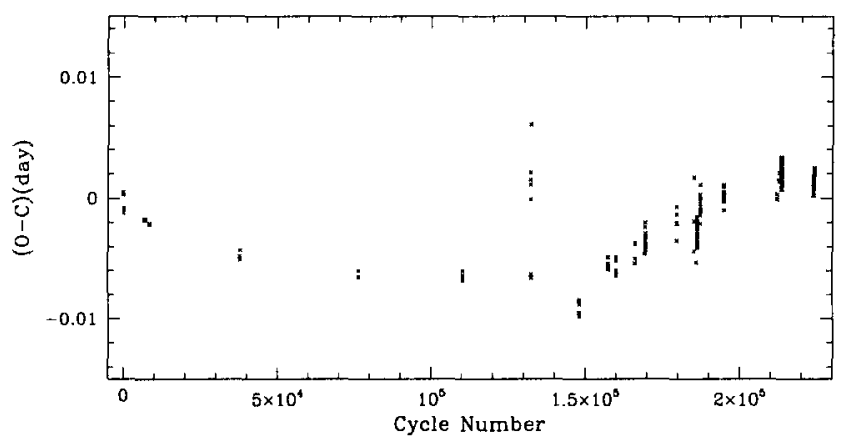

Figure 1. Difference between the observed times of maximum light and the times of maximum light calculated for a total of 240 points.

Table 1. Coefficients of the synthetic light curve of BL Cam.

\begin{tabular}{crcc}
\hline & \multicolumn{1}{c}{$f_{j}$} & $a_{j}$ & $\phi_{j}$ \\
\hline 1 & 25.5781 & 0.1115 & 1.1607 \\
2 & 51.1479 & 0.0367 & 1.2813 \\
3 & 25.5681 & 0.0361 & 1.3260 \\
4 & 1.9780 & 0.0090 & -1.6254 \\
5 & 31.6780 & 0.0083 & 2.8265 \\
6 & 76.7294 & 0.0065 & 1.7756 \\
7 & 25.2581 & 0.0063 & -0.7702 \\
8 & 32.1680 & 0.0046 & -1.4992 \\
9 & 51.4379 & 0.0047 & 0.4470 \\
$a_{0}$ & 0.9808 & & \\
r.m.s. & 0.0096 & & \\
$t_{0}=$ & HJD2450475.7864 & & \\
\hline
\end{tabular}

\section{References}

Kim, C., Sim, E.-J. 1999, J. Ast. Space Sci. (Korea), 16, 241 McNamara, D.H. \& Feltz, K.A. 1978, PASP, 90, 275 


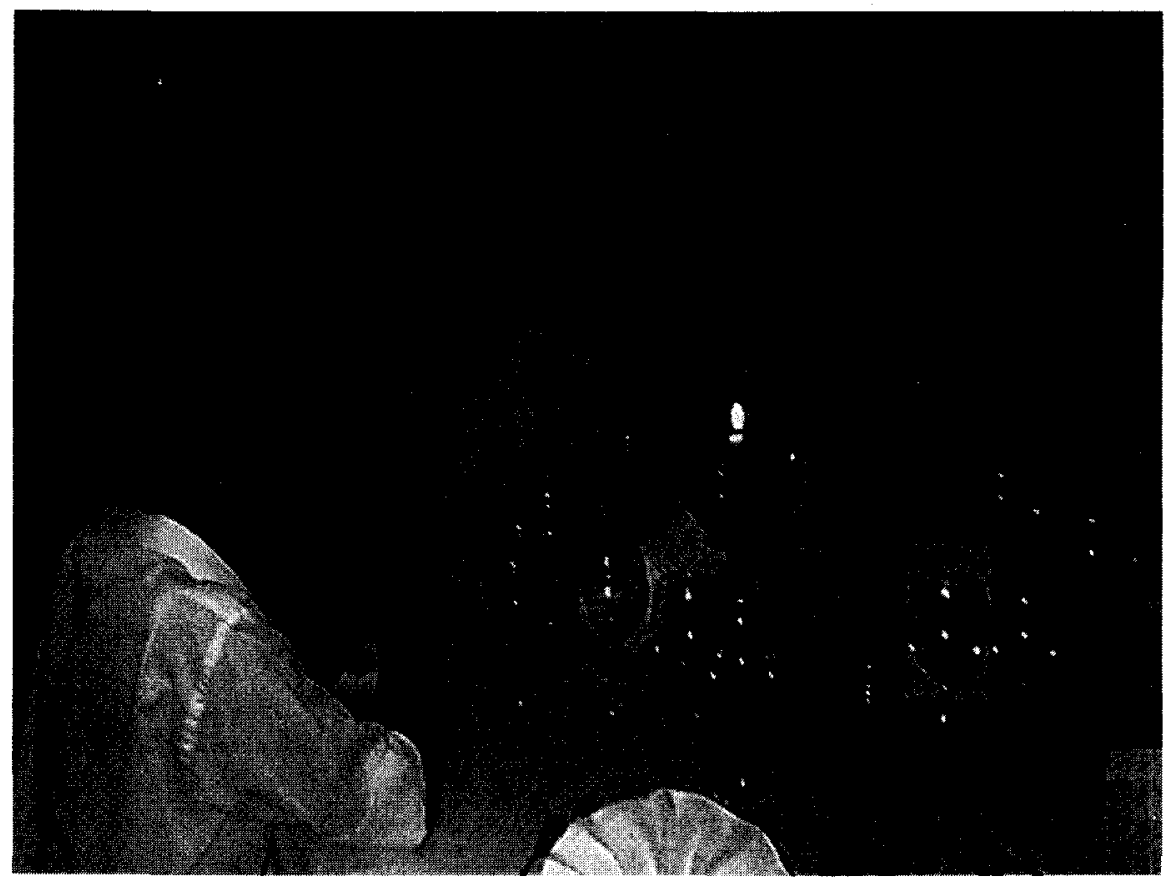

Erwin Sedlmayr is telling some young participants everything he knows about dust shells. 